\title{
ESTIMACIÓN DE NIVELES DE FONDO Y NIVELES DE REFERENCIA PARA HIERRO, MANGANESO, NÍQUEL Y ZINC EN SUELOS DE UN ÁREA NO INTERVENIDA ANTROPOGÉNICAMENTE
}

\author{
ESTIMATION OF BASE AND REFERENCE LEVELS FOR IRON, \\ MANGANESE, NICKEL AND ZINC IN SOILS OF AN AREA NOT INTERVENED \\ ANTHROPOGENICALLY
}

\begin{abstract}
Jorge Briceño ${ }^{1,2}$; Arnaldo Armado²; Ángela Sequera² y Zulay Niño-Ruiz ${ }^{3}$.
(I)Universidad Técnica de Ambato, Facultad de Ciencia e Ingeniería en Alimentos, Ambato - Ecuador. jbriceno@uc.edu.ve

(2) Universidad de Carabobo, Centro de Investigación en Ambiente, Biología y Química (AMBIOQUIM), Departamento de Química, Facultad Experimental de Ciencias y Tecnología, Valencia - Venezuela

(3) Universidad Regional Amazónica, IKIAM, Tena - Ecuador, Km 7 Vía Muyuna, Tena - Ecuador
\end{abstract} https://doi.org/10.33789/talentos.7.1.118

\begin{abstract}
Resumen: La determinación de metales en suelos es de suma importancia, ya que tiene aplicaciones en diferentes campos. En Venezuela, hasta la fecha no existe una norma que establezca los niveles basales de metales en suelos, que permita implementar indicadores para suelos sanos o no intervenidos. En ese sentido, se plantea determinar el contenido de Fe, Mn, Niy Zny algunas propiedades fisicoquímicas en suelos de zonas no intervenidas antropogénicamente, como lo es el suelo del Parque Municipal Casupo, zona protegida ubicada en Valencia al norte de Venezuela. El contenido de metales se cuantificó empleando Absorción Atómica con atomizador de llama con fuente de línea y deuterio para corregir la absorbancia inespecifica. Los suelos resultaron ser de arenosos a franco, con pH entre 4,5-7,6, la conductividad eléctrica se encontró por debajo de $500 \mathrm{mS} / \mathrm{cm}$ y la materia orgánica entre 2,8 y 7\%. El contenido de $\mathrm{Fe}, \mathrm{Mn}$, Ni y Zn se encontró entre 34-208, 32-205, 3-197 y 10-43 mg de metal/kg de suelo seco, respectivamente. Los niveles de fondo fueron determinados para Fe (119 mg/kg), Mn (108 mg/kg), Ni (12 mg/kg) y Zn (25 mg/kg). En base a estos, fueron propuestos como niveles de referencia 120, 194, 18 y $100 \mathrm{mg} / \mathrm{kg}$ para $\mathrm{Fe}$, Mn, Ni y Zn, respectivamente. Estos valores pueden ser empleados para proponer límites permisibles adecuados, de los metales en estudio en suelos y establecer normas para su uso apropiado.
\end{abstract}

Recibido: 17 de marzo de 2020

Aceptado: 21 de junio de 2020

Publicado como artículo de investigación en la Revista de Investigación Talentos VII (1) 1-11 
Palabras Clave: Metales pesados en suelos, niveles basales, niveles de referencia, Parque Municipal Casupo, suelos protegidos.

Abstract: The determination of metals in soils is of paramount importance, since it has applications in different fields. In Venezuela, there is no standard to date that establishes the basal levels of metals in soils, which allows the implementation of indicators for healthy or non-intervened soils. In that sense, it is proposed to determine the content of $\mathrm{Fe}, \mathrm{Mn}, \mathrm{Ni}$ and $\mathrm{Zn}$ and some physicochemical properties in soils with no anthropogenic intervention, such as the soils of the Casupo Municipal Park, a protected area located in Valencia, northern Venezuela. The metal content was quantified using Atomic Absorption with flame atomizer with line source and deuterium to correct nonspecific absorbance. The soils turned out to be sandy to loam, with pH between 4.5 and 7.6, the electrical conductivity was below $500 \mathrm{mS} / \mathrm{cm}$, and the organic matter between 2.8 and $7 \%$. The content of $\mathrm{Fe}, \mathrm{Mn}$, Ni and $\mathrm{Zn}$ was found to range from 34 to 208, 32 to 205, 3 to 197, and 10 to $43 \mathrm{mg}$ of metal / $\mathrm{kg}$ of dry soil, respectively. Base levels were determined for Fe (119 mg / kg), Mn (108 mg / kg), Ni (12 mg/ kg) and Zn (25 mg / kg). Based on these, reference levels of 120, 194, 18 and $100 \mathrm{mg} / \mathrm{kg}$ were proposed for Fe, Mn, Ni and Zn, respectively. These values can be used to propose appropriate permissible limits of the metals under study in soils and establish standards for their appropriate use.

Keywords: Heavy metals in soils, basal levels, reference levels, Casupo Municipal Park, protected soils.

\section{INTRODUCCIÓN}

El crecimiento poblacional mantiene constante presión sobre el uso de los suelos. Es nuestra responsabilidad hacer uso de manera sostenible, para mantener su integridad funcional o permitir su resiliencia, definida como el potencial para recuperar la integridad funcional y estructural después de una perturbación (Ludwig, Wilmes, \& Schrader, 2018).

Actualmente existe una preocupación mundial por conocer el contenido de metales en suelos debido a sus implicaciones de riesgos a la salud pública. Se han estudiado suelos superficiales de zonas urbanas empleando el índice de geoacumulación, el índice de contaminación y el índice de riesgo ecológico potencial para evaluar los niveles de contaminación por metales (Qing, Yutong, \& Shenggao, 2015) (Hu et al., 2013).

La importancia en el seguimiento de los metales pesados en suelos radica en que estos pueden trasladarse a los alimentos y finalmente llegar al consumo humano (Tóth, Hermann, Da Silva, \& Montanarella, 2016) generando diversas afecciones a la salud, incluso la muerte.

La incorporación de metales a las plantas se produce mediante factores que controlan su distribución y transferencia dentro de los 
sistemas suelo-vegetación que no siempre están bien definidos (Chopin \& Alloway, 2007).

Algunas investigaciones se basan en analizar metales totales y biodisponibles en zonas que han sido afectadas por algún medio contaminante externo, y se compara con el más lejano a la zona afectada, es importante tener un patrón de comparación que determine el nivel de contaminación que se tiene para determinados suelos (Briceño, Márquez, Sánchez, \& Armado, 2018).

El índice de contaminación resulta una herramienta ampliamente utilizada para la evaluación integral del grado de contaminación de un suelo. Es necesario determinar los valores de referencia de metales en los suelos, puesto que el índice de contaminación no es más que el resultado de dividir la concentración del metal en la zona de estudio por la concentración de referencia del mismo metal (Kowalska, Mazurek, Gąsiorek, \& Zaleski, 2018).

Por esta razón, en algunos países se han establecido niveles de referencia con rango normativo. Sin embargo, hasta la fecha en Venezuela no se tiene conocimiento de una norma que establezca los niveles de referencia de metales en suelos. Por lo que, es de interés conocer el contenido de metales en suelos en donde no hay intervención antropogénica, y de esta manera estimar los niveles de fondo de metales en suelos (niveles basales), que permitan establecer, dichos niveles de referencia (Brizuela \& Jiménez, 2012).

Considerando que la contaminación del suelo está íntimamente relacionada con el desarrollo de las actividades humanas, la definición de niveles de referencia para metales resulta particularmente importante para valorar tal contaminación.

Como se mencionó anteriormente, para establecer niveles de referencia, es necesario conocer los niveles basales de metales en suelos, lo que se puede lograr conociendo el contenido de metales en suelos no intervenidos antropogénicamente, es decir, zonas protegidas como Parques Nacionales, Parques Municipales, entre otras.

En este orden de ideas, Venezuela posee distintos parques Nacionales y zonas protegidas, específicamente, en la ciudad de Valencia, capital del estado Carabobo, se encuentra el Parque Municipal Casupo, área seleccionada para esta investigación, en donde se realizó el estudio del contenido de hierro, manganeso, níquel y zinc, con el objetivo de estimar los niveles basales y de referencia de estos metales como parámetro indicador de suelos sanos o no intervenidos lo que serviría de comparación con suelos potencialmente contaminados.

\section{MATERIALES Y MÉTODOS}

\section{Muestreo de suelos}

Las muestras de suelo se recolectaron en sitios de mínimo impacto antropogénico. Con la ayuda de los guardabosques fue posible acceder por sitios alejados de las caminerías. En la Fig. 1, señalada con un círculo rojo se muestra el área muestreada, tomando en cuenta la accesibilidad y las condiciones de la zona. Se tomaron treinta y dos muestras, 
para lo cual se utilizó el método aleatorio estratificado, dentro de los distintos lugares fijados se excavó de 10-30 cm de profundidad tomando 1-2 kg de suelo aproximadamente. Las muestras fueron trasladadas al laboratorio en bolsas de polietileno limpias debidamente etiquetadas y almacenadas adecuadamente hasta su análisis. Las muestras de suelo fueron secadas a temperatura ambiente, molidas empleando un mortero y pistillo de cerámica y finalmente tamizadas con una malla No. 14 (Faithfull \& Ferrando Navarro, 2005).

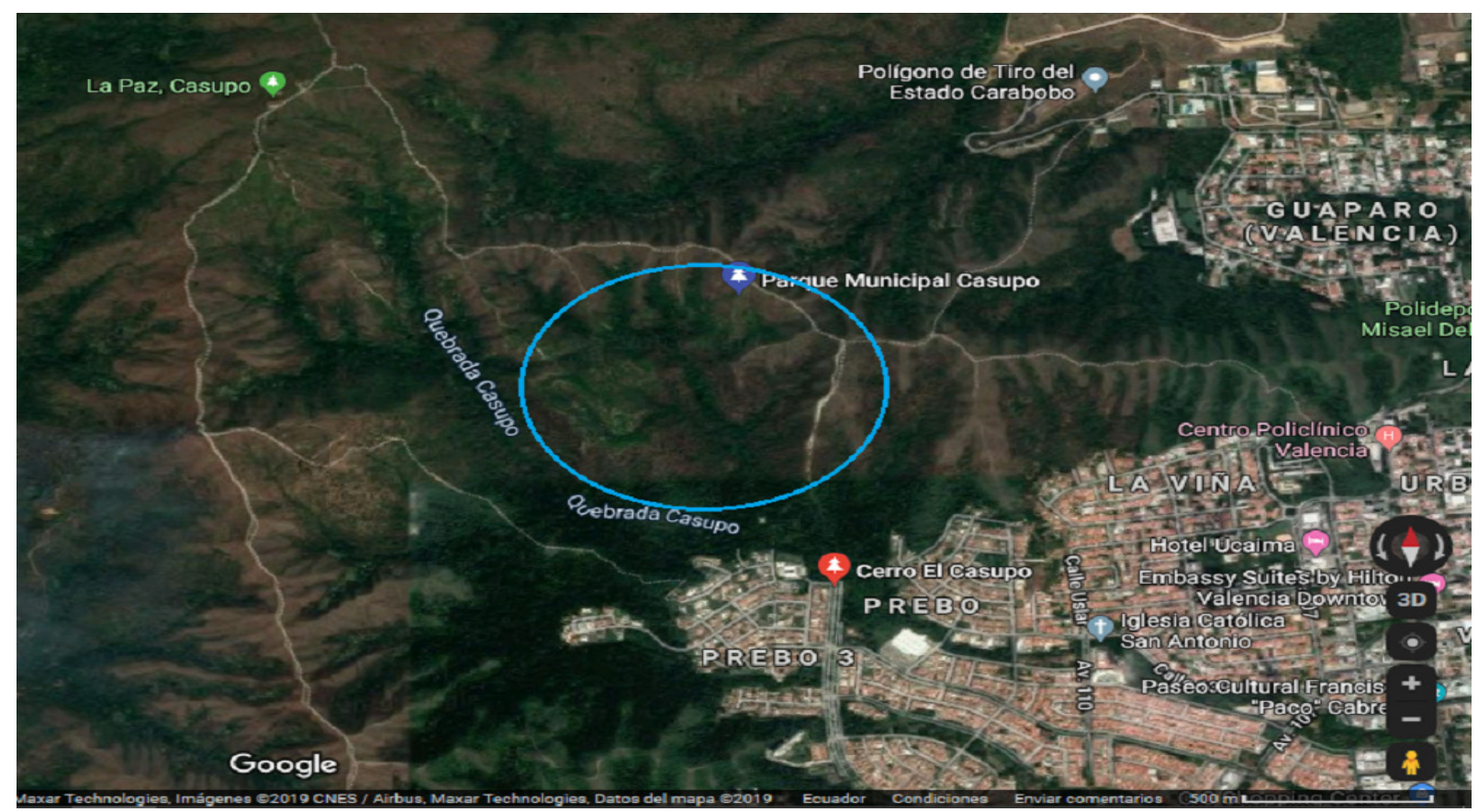

Fig. 1. Imagen aérea de la zona objeto de estudio. Parque Municipal Casupo, Estado Carabobo, Venezuela. (Imágenes.googleMaps.2019)

\section{Parámetros fisicoquímicos}

El contenido de humedad del suelo se determinó por pérdida de peso en una estufa utilizando el método 93.06-37.1.10 (AOAC, 2006), y posteriormente el contenido de la materia orgánica por diferencia de peso entre la masa inicial de la muestra secada a $105^{\circ} \mathrm{C}$ y la masa después de ser calcinada a $430{ }^{\circ} \mathrm{C}$ por $10 \mathrm{~h}$ (Cargua Catagña, Rodríguez Llerena, Damián Carrión, Recalde Moreno, \& Santillán Lima, 2017). En ambos casos se empleó una mufla NABERTHERM LT 15/12/B180.

El pH y la conductividad eléctrica del suelo se determinaron a través de un extracto preparado con suelo y agua bidestilada en proporción $1: 2,5(\mathrm{p} / \mathrm{v})$ con agitación y reposo de $60 \mathrm{~min}$ (Kazlauskaite-Jadzeviče, Volungevičius, Gregorauskiene, \& Marcinkonis, 2014). Se pesó $3 \mathrm{~g}$ de suelo por triplicado y se adicionó $6 \mathrm{ml}$ de agua bidestilada en un tubo tipo Falcon, se colocó en un agitador orbital por 30 minutos y posteriormente fueron centrifugadas por $5 \mathrm{~min}$ a $3000 \mathrm{rpm}$ y se determinó $\mathrm{pH}$ y conductividad al sobrenadante. La textura se estimó mediante el método de dispersión-sedimentación (Di Stefano, Ferro, \& Mirabile, 2010). 


\section{Preparación de las muestras previo a la determinación de los metales}

La estimación de los metales totales en suelo se hizo mediante digestión con Agua Regia recién preparada $\left(\mathrm{HNO}_{3}\right.$ concentrado y $\mathrm{HCl}$ concentrado en proporción 1:3 v/v), para ello se pesó $0,5 \mathrm{~g}$ de suelo previamente tamizado en una balanza analítica Mettler Toledo modelo XPE204 y se agregó el Agua Regia en una proporción 1:10 p/v, el sistema se colocó a $90{ }^{\circ} \mathrm{C}$ por $2 \mathrm{~h}$ con agitación magnética, posteriormente se filtró y transfirió cuantitativamente a un matraz aforado de $25 \mathrm{ml}$ con $\mathrm{HNO}_{3}$ 0,14 M (Golia, Dimirkou, \& Mitsios, 2008).

\section{Determinación del contenido de metales}

La determinación de los metales se hizo con un espectrofotómetro de absorción atómica con fuente de línea Thermo Scientific modelo SOLAAR Serie $\mathrm{S}$ equipado con lámpara de cátodo hueco como fuente de radiación y $\mathrm{D}_{2}$ como sistema de corrección de la absorbancia inespecífica. Para la llama se utilizó acetileno grado 2,5 y pureza 99,5\% (Oxicar Venezuela).

Diariamente fueron preparadas soluciones patrones de zinc, hierro, manganeso y níquel a partir de sus respectivos patrones acuosos monoelementales (AccuStandart) de $1 \mathrm{~g} / \mathrm{L}$ de cada metal. Los patrones fueron diluidos con ácido nítrico 0,14 M (Merck). El intervalo de concentración de los patrones se muestra en la Tabla I y se emplearon las condiciones instrumentales establecidas por el fabricante.
Tabla I. Intervalo de concentración y longitud de onda para la determinación de metales mediante espectrometría de absorción atómica a la llama.

\begin{tabular}{ccc}
\hline Metal & $\begin{array}{c}\text { Longitud de } \\
\text { onda (nm) }\end{array}$ & $\begin{array}{c}\text { Intervalo de } \\
\text { concentración } \\
\left(\mathbf{m g} \cdot \mathbf{L}^{-\mathbf{1}}\right)\end{array}$ \\
\hline Zinc & 213,9 & $0,05-2$ \\
Hierro & 248,3 & $0,3-10$ \\
Manganeso & 279,5 & $0,1-4$ \\
Níquel & 232,0 & $0,3-8$ \\
\hline
\end{tabular}

En todos los dos casos la cuantificación del contenido de metales se hizo con el método de calibración con estándar externo mediante comparación directa de la señal de cada elemento frente al calibrado obtenido para cada metal (Faithfull \& Ferrando Navarro, 2005). Todas las muestras se procesaron por triplicado, incluyendo un blanco intercalado entre cada muestra. La calidad de los resultados se verificó empleando un punto de calibración preparado de manera independiente encontrándose en todos los casos valores dentro de la ventana de aceptación.

\section{Estimación de niveles de fondo y niveles} genéricos de referencia

Para proponer los niveles de fondo (NF) y niveles genéricos de referencia (NR) se utilizó la metodología reportada por Brizuela y Jiménez (2012). En resumen, se realiza una prueba de normalidad y para los datos cuya distribución resulte normal, el NF es la media aritmética (promedio). Los niveles genéricos de referencia (NR) se expresan en función a los NF y la desviación estándar 
(DE) (Ecuación 1). En el caso de encontrarse correlación del contenido de metales con los parámetros edáficos determinados, se debe usar la ecuación 2.

$\mathrm{NR}=\mathrm{NF}+2 \mathrm{DE}$

$\mathrm{NR}=\mathrm{NF}+\mathrm{Aa}+\mathrm{Bb}+\ldots+\mathrm{Zz}$

Donde A, B, ..., Z son los valores medios de los parámetros edáficos; $\mathrm{a}, \mathrm{b}, \ldots, \mathrm{z}$ son las pendientes de las rectas de regresión lineal simple.

En el caso de que no resulte una distribución normal o es log-normal y su desviación estándar (DE) es pequeña se toma la media geométrica (mediana) como nivel de fondo, pero si la desviación estándar es alta el NF se expresa en función a la diferencia intercuartiles $\left(3^{\circ} \mathrm{I}-1^{\circ} \mathrm{I}\right)$. En este caso el NR se determina mediante la ecuación 3.

$\mathrm{NR}=\left(3^{\circ} \mathrm{I}-1^{\circ} \mathrm{I}\right) * 1,5$

Todos los análisis estadísticos se realizaron con el programa PAST3 versión 3.12 (Hammer, 2016).

\section{RESULTADOS Y DISCUSIÓN}

Propiedades fisicoquímicas de suelos del Parque Municipal Casupo

En la Figura 2 se señala el promedio con línea de trazo continuo color verde, mientras que las bandas de confianza se muestran en función de la desviación estándar, empleando líneas discontinuas azul y rojo para $\pm 2 \mathrm{~s} \mathrm{y} \pm 3 \mathrm{~s}$ respectivamente.

Los valores de $\mathrm{pH}$ encontrados en suelos del parque Municipal Casupo se muestran en la Figura 2A. Estos valores de $\mathrm{pH}$ (promedio $5,9)$ se corresponden con suelos que van desde fuertemente ácidos $(4,5)$ hasta mediamente alcalino $(7,5)$ según la clasificación de la Secretaría de Medio Ambiente y Recursos Naturales de Ecuador (SEMARNAT, 2003).

En cuanto a los valores encontrados de conductividad eléctrica se muestran en la Figura 2B. En todos las zonas muestreadas presentaron valores por debajo de $1000 \mathrm{mS} /$ $\mathrm{cm}$ correspondiente a un suelo con efecto despreciable de salinidad y la materia orgánica (Figura 2C) estuvo con valores entre bajo y medio (SEMARNAT, 2003). En la estimación de la textura, el contenido de arena se encontró entre 30-100 \%, limo entre 0-32 $\%$ y arcilla entre $0-60 \%$, resultando clases texturales que van desde arenosa a franca.

Contenido de hierro, manganeso, níquel y zinc en suelos del Parque Municipal Casupo.

Los resultados obtenidos para los metales $\mathrm{Fe}, \mathrm{Mn}, \mathrm{Ni}$ y Zn, en los suelos del Parque Municipal Casupo se presentan en la Tabla II. Se muestran los valores mínimos y máximos, valores promedio, mediana, desviación estándar, entre otros estadísticos descriptivos que permitieron el cálculo de los niveles de fondo y niveles de referencia propuestos.

El contenido de hierro se encontró entre 34 y $208 \mathrm{mg} / \mathrm{kg}$ de suelo, con un promedio de $119 \mathrm{mg} / \mathrm{kg}$, teniendo una distribución normal (aplicando las pruebas de normalidad Shapiro Wilk W. y Jarque-Gera JB). Este intervalo determinado se asemeja al encontrado por Noguera y Armado (2010) en suelos impactados y no impactados con petróleo en Yaracal, estado Falcón, Venezuela, que reportan valores entre $38-153 \mathrm{mg} / \mathrm{kg}$. Sin embargo, están muy por debajo de los 
obtenidos por el Instituto Geológico y Minero de España (Raimundo Jiménez Ballesta, 2017) que reportan valores desde 300 hasta 6730 mg/kg en la comunidad de Aragón.

El contenido de manganeso varió entre 32 y $205 \mathrm{mg} / \mathrm{kg}$ de suelo, con un promedio de $108 \mathrm{mg} / \mathrm{kg}$, teniendo una distribución normal (aplicando las pruebas de normalidad Shapiro Wilk W. y Jarque-Gera JB). Valores similares $(5-156 \mathrm{mg} / \mathrm{kg})$ son reportados en suelos de Yaracal, estado Falcón, Venezuela (Noguera \& Armado, 2010). En España, en la comunidad de Aragón se reportan valores de $\mathrm{Mn}$ entre 39 y $1750 \mathrm{mg} / \mathrm{kg}$ (Raimundo Jiménez Ballesta, 2017).

En el caso del $\mathrm{Ni}$, los valores obtenidos estuvieron entre 3 y $197 \mathrm{mg} / \mathrm{kg}$, con una media geométrica (mediana) de $10 \mathrm{mg} / \mathrm{kg}$ con una distribución no normal (aplicando las pruebas de normalidad Shapiro Wilk W. y Jarque-Gera JB). Intervalos de 11-109 y de 3-122 mg/kg fueron reportados para $\mathrm{Ni}$, en suelos de Venezuela (Noguera \& Armado, 2010) y España (Raimundo Jiménez Ballesta, 2017), respectivamente.

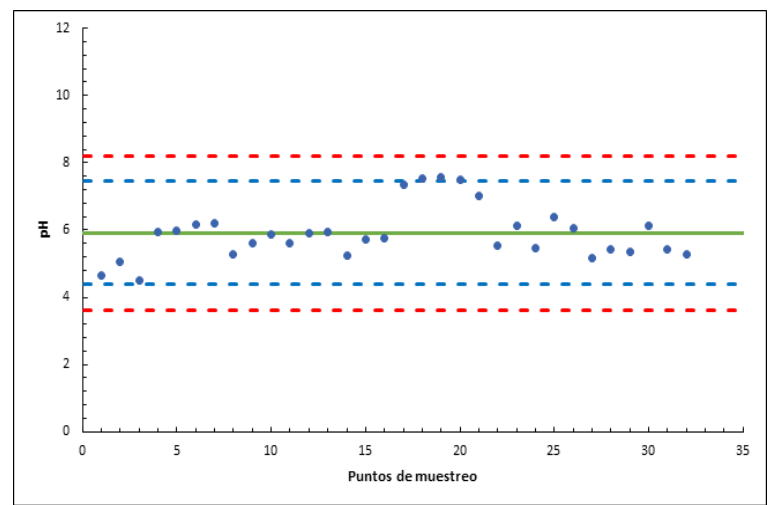

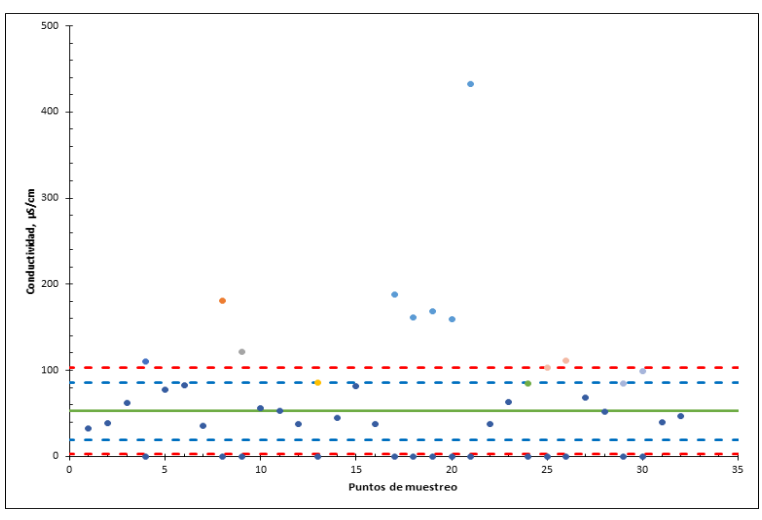

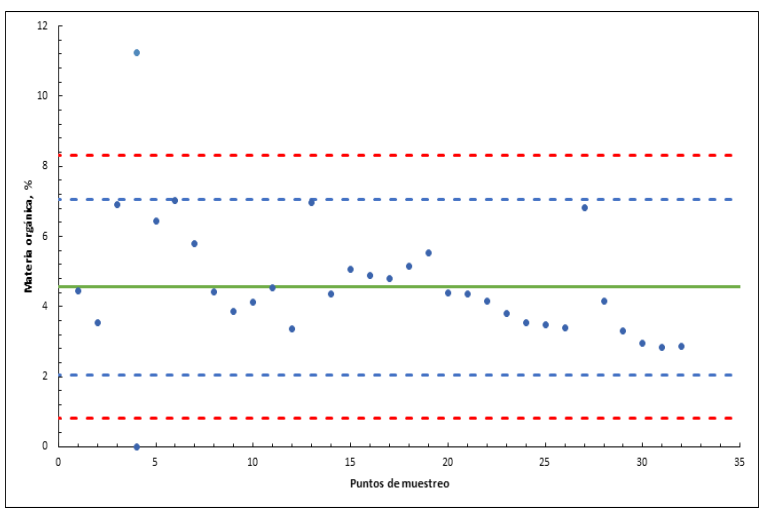

Fig. 2. Propiedades del suelo estudiado. Potencial de hidrógeno $\mathrm{A}$, conductividad $\mathrm{B}$ y materia orgánica $\mathrm{C}$ en suelos del parque Municipal Casupo. - Media $- \pm 2 \mathrm{~s}$; $- \pm 3 \mathrm{~s}$

Para el $\mathrm{Zn}$, los valores obtenidos van desde 10 a $43 \mathrm{mg} / \mathrm{kg}$, con un promedio de $25 \mathrm{mg} / \mathrm{kg}$, teniendo una distribución normal (aplicando las pruebas de normalidad Shapiro Wilk W. y Jarque-Gera JB). Estos valores en el contenido de Zn se encuentran dentro de los intervalos de 11-109 mg/kg reportados para suelos venezolanos (Noguera \& Armado, 2010) y de 3-122 mg/kg para suelos de España (Raimundo Jiménez Ballesta, 2017).

Niveles basales y niveles de referencia.

Los resultados conseguidos para los niveles de fondo y niveles de referencia se muestran en la Tabla II los cuales fueron determinados empleando las Ecuaciones 1 y 2. 
Tabla II. Estadísticos descriptivos, niveles de fondo y niveles de referencia para Fe, Mn, Ni y Zn en suelos del parque municipal casupo

\begin{tabular}{lcccc} 
& \multicolumn{4}{c}{ Niveles de los metales en el suelo (mg/kg) } \\
& Fe & Mn & Ni & Zn \\
\hline Mínimo & 34 & 32 & 3 & 10 \\
Máximo & 208 & 205 & 197 & 43 \\
Promedio & 119 & 108 & 21 & 25 \\
Desviación estándar & 47 & 43 & 35 & 10 \\
Mediana & 114 & 93 & 10 & 22 \\
Percentil 25 (primer intercuartil) & 78 & 75 & 7 & 17 \\
Percentil 75 (tercer intercuartil) & 151 & 140 & 19 & 33 \\
Niveles de Fondo & $\mathbf{1 1 9}$ & $\mathbf{1 0 8}$ & $\mathbf{1 2}$ & $\mathbf{2 5}$ \\
Niveles de referencia & $\mathbf{1 2 0}$ & $\mathbf{1 9 4}$ & $\mathbf{1 8}$ & $\mathbf{1 0 0}$ \\
\hline
\end{tabular}

Promedio, mínimo y máximo para $n=32$.

En base al análisis estadístico, los niveles de fondo para Fe (119 mg/kg), Mn (108 mg/ $\mathrm{kg})$ y Zn $(25 \mathrm{mg} / \mathrm{kg})$ son igual a su media aritmética (promedio), ya que presentan un comportamiento normal; en el caso del Ni presentó un comportamiento no normal y desviación estándar elevada, por lo que el nivel de fondo (12 mg/kg) se basa en la diferencia intercaurtiles.

Para los niveles de referencia basados en los niveles de fondo, para el Manganeso, se propone un nivel de referencia genérico de $194 \mathrm{mg} / \mathrm{kg}$, empleando la ecuación 1. Los niveles de referencia propuestos para el Fe $(120 \mathrm{mg} / \mathrm{kg})$ y Zn $(100 \mathrm{mg} / \mathrm{kg})$ se calcularon mediante la ecuación 2, teniendo en consideración que el contenido de Fe presentó correlación significativa $(\mathrm{p}<0,05)$ inversa con el porcentaje de limo; y el contenido de $\mathrm{Zn}$ presentó correlación significativa $(\mathrm{p}<0,05)$ directa con el porcentaje de arena, e inversa con el porcentaje de arcilla. Para el Ni el nivel de referencia fue $18 \mathrm{mg} / \mathrm{kg}$ y se calculó usando la ecuación 3.
Los niveles de fondo $y$ de referencia propuestos en esta investigación, en general, se encuentran por debajo de los valores reportados para los metales en estudio por otros autores. En un estudio similar se reporta para Fe, valores NF de $2020 \mathrm{mg} / \mathrm{kg}$ y NR entre 3750-10000 mg/kg; Mn, los NF de 359 mg/ kg y NR entre 715-1335 mg/kg; Ni valores NF de 19 mg/kg y NR entre 40 y 75 mg/kg; y Zn con valores de NF de $39 \mathrm{mg} / \mathrm{kg}$ y NR entre 130-395 mg/kg (Raimundo Jiménez Ballesta, 2017). Por su parte, Jiménez Ballesta et al., (2010) reportan para $\mathrm{Ni}$, valores de 20,81 y $42,6 \mathrm{mg} / \mathrm{kg}$; para $\mathrm{Zn}$, valores de 32,19 y 86,5 $\mathrm{mg} / \mathrm{kg}$ de los NF y NR, respectivamente.

Como puede observarse, los valores de NF y NR reportados por otros investigadores para Fe y Mn son muy elevados, respecto a los propuestos en este trabajo. En el caso de Ni y Zn, reportan valores dentro del intervalo obtenido.

Los niveles de referencia propuestos en esta investigación (Tabla II), pueden ser la base para proponer límites permisibles 
adecuados, para los metales en estudio (Fe, $\mathrm{Mn}, \mathrm{Ni}$ y $\mathrm{Zn}$ ) en suelos con características similares al Parque Municipal Casupo, que sean intervenidos antropológicamente, para considerar si se presenta contaminación o no, frente a un eventual impacto ambiental. Cabe destacar que, aunque Venezuela presenta un marco legal amplio en políticas ambientales, no hay normativas específicas que regulen el impacto de metales en suelos, por actividades antrópicas (Brizuela \& Jiménez, 2012).

\section{CONCLUSIONES}

Los suelos seleccionados del Parque Municipal Casupo resultaron ser arenosos a franco, con un potencial de hidrógeno desde fuertemente ácido hasta mediamente alcalino, con efectos despreciables de salinidad y de bajo a medio el contenido de materia orgánica. El contenido de Fe y Mn en los suelos fue muy bajo respecto a los valores reportados generalmente para diferentes suelos. El contenido de Ni y Zn se encontró dentro de los valores reportados por otros investigadores.

Se estimó que los niveles de fondo para los suelos del Parque Municipal Casupo son para el Fe, 119 mg/kg; Mn, 108 mg/kg; Ni, $12 \mathrm{mg} / \mathrm{kg}$ y Zn, $25 \mathrm{mg} / \mathrm{kg}$. Se proponen como niveles de referencia los siguientes valores 120, 194, 18 y 100 mg/kg para Fe, Mn, Ni y $\mathrm{Zn}$ respectivamente, los cuales permitirían orientar normativas específicas, para regular el impacto antropogénico en suelos venezolanos.

\section{AGRADECIMIENTOS}

Este trabajo ha sido financiado por la DIDEUTA a través del proyecto Biodisponibilidad de metales en suelos del cantón Quero de la provincia de Tungurahua HCU 0194-CU-P2018 y el Consejo de Desarrollo Científico y Humanístico de la Universidad de Carabobo, mediante el proyecto $\mathrm{CDCH}-2010-005$.

\section{REFERENCIAS}

AOAC. (2006). Official methods of analysis Proximate Analysis and Calculations Moisture (M) Fruits, Vegetables, and their Products - item 107. Association of Analytical Communities. Reference data: Method 934.06 (37.1.10); NFNAP; WATER.

Briceño, J., Márquez, M., Sánchez, K., \& Armado, A. (2018). Lignite as organic amendment in a dross affected soils. Italian Journal of Food Science, 31(5), 164-170.

Brizuela, J., \& Jiménez, Y. (2012). Metodologías aplicadas para el establecimiento de los niveles de referencia para metales pesados en la evaluación de la contaminación en suelos. Avances En Quimica, 7(2), 101109.

Cargua Catagña, F. E., Rodríguez Llerena, M. V., Damián Carrión, D. A., Recalde Moreno, C. G., \& Santillán Lima, G. P. (2017). Analytical methods comparison for soil organic carbon determination in Andean Forest of Sangay National Park-Ecuador. Acta Agronómica, 66(3), 408-413. https://doi.org/10.15446/acag. v66n3.52467

Chopin, E. I. B., \& Alloway, B. J. (2007). Distribution and mobility of trace 
elements in soils and vegetation around the mining and smelting areas of Tharsis, Ríotinto and Huelva, Iberian Pyrite Belt, SW Spain. Water, Air, and Soil Pollution, 182(1-4), 245-261. https:// doi.org/10.1007/s11270-007-9336-X

Di Stefano, C., Ferro, V., \& Mirabile, S. (2010). Comparison between grainsize analyses using laser diffraction and sedimentation methods. Biosystems Engineering, 106(2), 205-215. https://doi.org/10.1016/j. biosystemseng.2010.03.013

Faithfull, N. T. (Nigel T. ., \& Ferrando Navarro, A. C. (2005). Métodos análisis químico agrícola: manual práctico. Acribia. Retrieved from https://www. editorialacribia.com/libro/metodosde-analisis-quimico-agricola-manualpractico_54383/

Golia, E. E., Dimirkou, A., \& Mitsios, I. K. (2008). Influence of Some Soil Parameters on Heavy Metals Accumulation by Vegetables Grown in Agricultural Soils of Different Soil Orders. Bulletin of Environmental Contamination and Toxicology, 81(1), 80-84. https://doi.org/10.1007/s00128008-9416-7

Hammer, Ø. (2016). PAST PAleontological STatistics Version 3.12 Reference manual Natural History Museum University of Oslo, (1999). Retrieved from http://folk.uio.no/ohammer/past

Hu, Y., Liu, X., Bai, J., Shih, K., Zeng, E. Y., \& Cheng, H. (2013). Assessing heavy metal pollution in the surface soils of a region that had undergone three decades of intense industrialization and urbanization. Environmental Science and Pollution Research, 20(9), 61506159. https://doi.org/10.1007/s11356013-1668-z

Jiménez Ballesta, R., Conde Bueno, P., Martín Rubí, J. A., \& García Giménez, R. (2010). Niveles de fondo geoquímico e influencia del marco geológico en las concentraciones edafogeoquímicas de base de suelos seleccionados de CastillaLa Mancha. Estudios Geologicos, 66(1), 123-130. https://doi.org/10.3989/ egeol.40214.119

Jiménez Ballesta, Raimundo. (2017). Introducción a la contaminación de suelos. (Mundi-Prensa, Ed.). Madrid.

Kazlauskaite-Jadzeviče, A., Volungevičius, J., Gregorauskiene, V., \& Marcinkonis, S. (2014). The role of $\mathrm{pH}$ in heavy metal contamination of urban soil. Journal of Environmental Engineering and Landscape Management, 22(4), 311318. https://doi.org/10.3846/16486897. 2013.872117

Kowalska, J. B., Mazurek, R., Gąsiorek, M., \& Zaleski, T. (2018). Pollution indices as useful tools for the comprehensive evaluation of the degree of soil contamination-A review. Environmental Geochemistry and Health, 40(6), 23952420. https://doi.org/10.1007/s10653018-0106-z

Noguera, S., \&Armado,A. (2010). Evaluación de metales en suelos contaminados por derrames de crudo en Yaracal, estado 
Falcón, Venezuela. Avances En Química, 5(3), 167-175.

Qing, X., Yutong, Z., \& Shenggao, L. (2015).

Assessment of heavy metal pollution and human health risk in urban soils of steel industrial city (Anshan), Liaoning, Northeast China. Ecotoxicology and Environmental Safety, 120, 377-385. https://doi.org/10.1016/j. ecoenv.2015.06.019

SEMARNAT. (2003). Acuerdo que establece las reglas de operación para el otorgamiento de Pagos del Programa de Servicios Ambientales Hidrológicos. Viernes, 3, 6-23. SGR. (2014). Programa de Prevención y Mitigación para Reducir el Riesgo por Diferentes Amenazas. Secretaría de Medio Ambiente y Recursos Naturales.

Tóth, G., Hermann, T., Da Silva, M. R., \& Montanarella, L. (2016). Heavy metals in agricultural soils of the European Union with implications for food safety. Environment International, 88, 299-309. https://doi.org/10.1016/j. envint.2015.12.017 